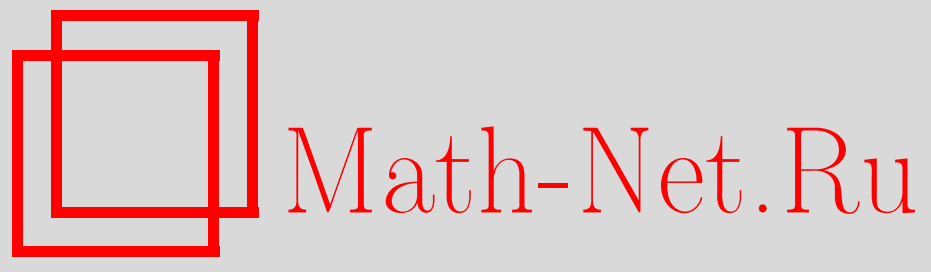

Р. Джакив, Симметрия Вейля и теория Лиувилля, $Т M \Phi$, 2006, том 148, номер 1, 80-88

DOI: https://doi.org/10.4213/tmf2059

Использование Общероссийского математического портала Math-Net.Ru подразумевает, что вы прочитали и согласны с пользовательским соглашением http://www . mathnet.ru/rus/agreement

Параметры загрузки:

IP : 3.93 .64 .190

26 апреля 2023 г., 10:21:41

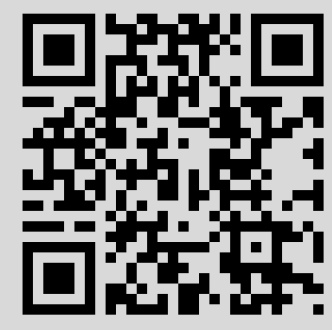




\section{СИММЕТРИЯ ВЕЙЛЯ И ТЕОРИЯ ЛИУВИЛЛЯ}

Конформная инвариантность в плоском пространстве и инвариантность Вейля в искривленном пространстве простым образом связаны друг с другом в размерности больше двух. Рассмотрена особая ситуация - теория Лиувилля в размерности два.

Ключевые слова: теория Лиувилля, преобразования Вейля, локальность, действие.

\section{1. ПОСВЯЩЕНИЕ}

Я познакомился с профессором Юрием Новожиловым в декабре 1971 г. на конференции по математической физике, которая проводилась в Физическом институте им. П.Н. Лебедева в Москве. С тех пор я несколько раз был его гостем в Ленинграде/Санкт-Петербурге, последний раз в прошлом году на Школе Фока, проводившейся при поддержке UNESCO, где я читал лекции по динамике, инвариантной относительно преобразований Вейля. По случаю замечательного юбилея Ю. Новожилова я рад представить ему эту работу, посвященную инвариантности Вейля в размерности два и ее связи с конформной инвариантностью и вейлевскими/конформными симметриями в высших размерностях.

\section{2. КОНФОРМНАЯ И ВЕЙЛЬ-ИНВАРИАНТНАЯ ДИНАМИКА СКАЛЯРНОГО ПОЛЯ ПРИ $d>2$}

Начнем с того, что запишем $d$-мерную лагранжеву плотность для скалярного поля $\varphi$ с масштабно- и конформно-инвариантным самодействием

$$
\mathcal{L}_{0}=\frac{1}{2} \eta^{\mu \nu} \partial_{\mu} \varphi \partial_{\nu} \varphi-\lambda \varphi^{2 d /(d-2)} .
$$

Очевидно, что это выражение имеет смысл только при $d \neq 2$, мы будем считать, что $d>2$. Теория инвариантна относительно преобразований

$$
\delta \varphi=f^{\alpha} \partial_{\alpha} \varphi+\frac{d-2}{2 d} \partial_{\alpha} f^{\alpha} \varphi
$$

${ }^{*}$ Center for Theoretical Physics Department of Physics, Massachusetts Institute of Technology Cambridge, MA, USA. E-mail: jackiw@lns.mit.edu 
где $f^{\alpha}$ - конформный вектор Киллинга (в плоском пространстве). Обычный канонический тензор энергии-импульса

$$
\theta_{\mu \nu}^{\text {canonical }}=\partial_{\mu} \varphi \partial_{\nu} \varphi-\eta_{\mu \nu}\left(\frac{1}{2} \eta^{\alpha \beta} \partial_{\alpha} \varphi \partial_{\beta} \varphi-\lambda \varphi^{2 d /(d-2)}\right)
$$

сохраняется и является симметричным, как это и должно быть в пуанкаре-инвариантной теории. Однако он не является бесследовым: $\eta^{\mu \nu} \theta_{\mu \nu}^{\text {canonical }} \neq 0$. Тем не менее, вследствие конформной инвариантности (2.2) тензор $\theta_{\mu \nu}^{\text {canonical }}$ можно улучшить путем добавления еще одного сохраняющегося и симметричного выражения, так что новый тензор становится бесследовым [1]:

$$
\theta_{\mu \nu}=\theta_{\mu \nu}^{\text {canonical }}+\frac{d-2}{4(d-1)}\left(\eta_{\mu \nu} \square-\partial_{\mu} \partial_{\nu}\right) \varphi^{2}, \quad \eta^{\mu \nu} \theta_{\mu \nu}=0 .
$$

Вариационный вывод канонического тензора (2.3) становится возможным после того, как в теорию (2.1) вводится минимальное взаимодействие с метрическим тензором $g_{\mu \nu}$, а интеграл действия варьируется по $g^{\mu \nu}$. Тензор $\theta_{\mu \nu}^{\text {canonical }}$ воспроизводится в пределе $g_{\mu \nu} \rightarrow \eta_{\mu \nu}$. Можно также аналогичным образом вывести улучшенный тензор (2.4), если выражение (2.1), обобщенное на случай искривленного пространства, расширить посредством специального неминимального взаимодействия [1]:

$$
\begin{aligned}
\mathcal{L}= & \frac{d-2}{8(d-1)} R \varphi^{2}+\frac{1}{2} g^{\mu \nu} \partial_{\mu} \varphi \partial_{\nu} \varphi-\lambda \varphi^{2 d /(d-2)} \\
T_{\mu \nu}= & \frac{2}{\sqrt{|g|}} \frac{\delta}{\delta g^{\mu \nu}} \int \sqrt{|g|} \mathcal{L}=\partial_{\mu} \varphi \partial_{\nu} \varphi-g_{\mu \nu}\left(\frac{1}{2} g^{\alpha \beta} \partial_{\alpha} \varphi \partial_{\beta} \varphi-\lambda \varphi^{2 d /(d-2)}\right)+ \\
& \quad+\frac{d-2}{4(d-1)}\left(g_{\mu \nu} D^{2}-D_{\mu} D_{\nu}+G_{\mu \nu}\right) \varphi^{2}
\end{aligned}
$$

Здесь $G_{\mu \nu}$ - тензор Эйнштейна, $R$ - скаляр Риччи, $R=2 /(2-d) g^{\mu \nu} G_{\mu \nu}$, а $D_{\mu}-$ ковариантная производная. В пределе $g_{\mu \nu} \rightarrow \eta_{\mu \nu}$ неминимальный член в выражении для $\mathcal{L}$ обращается в нуль, однако в вариации $g^{\mu \nu}$ он сохраняется:

$$
T_{\mu \nu} \underset{g_{\mu \nu} \rightarrow \eta_{\mu \nu}}{\longrightarrow} \theta_{\mu \nu}
$$

Заметим, что $g^{\mu \nu} T_{\mu \nu}=0$ в силу полевого уравнения для $\varphi$

$$
D^{2} \varphi+\lambda \frac{2 d}{d-1} \varphi^{(d+2) /(d-2)}-\frac{d-2}{4(d-1)} R \varphi=0 .
$$

Это обеспечивает обращение в нуль величины $\eta^{\mu \nu} \theta_{\mu \nu}$.

Точное выражение для неминимального взаимодействия приводит к инвариантности действия в искривленном пространстве относительно преобразований Вейля, включающих произвольную функцию $\sigma[2]$ :

$$
\begin{aligned}
g_{\mu \nu} & \rightarrow e^{2 \sigma} g_{\mu \nu}, \\
\varphi & \rightarrow e^{(2-d) \sigma / 2} \varphi .
\end{aligned}
$$


Самодействие инвариантно относительно преобразований (2.9). При этом кинетический член и член, связанный с неминимальным взаимодействием, не инвариантны по отдельности, однако их нетривиальные вариации при преобразованиях Вейля сокращаются при суммировании. Из инвариантности действия относительно преобразования Вейля также следует бесследовость его $g^{\mu \nu}$-вариации, т.е. бесследовость $T_{\mu \nu}$, равно как его инвариантность относительно диффеоморфизмов, обеспечивает симметрию и ковариантное сохранение $T_{\mu \nu}$.

Таким образом, мы видим, что инвариантность относительно преобразований Вейля (и относительно диффеоморфизмов) в искривленном пространстве тесно связана с конформной инвариантностью в плоском пространстве [2]. Однако всегда ли конформно-инвариантную теорию в плоском пространстве можно расширить до теории, инвариантной относительно преобразований Вейля и относительно диффеоморфизмов в искривленном пространстве? Очевидно, ответ будет положительным для самовзаимодействующих скалярных теорий при $d>2$, обсуждавшихся в предыдущей работе [3]. Теперь посмотрим, что происходит при $d=2$.

\section{3. ТЕОРИЯ ЛИУВИЛЛЯ: ДИНАМИКА КОНФОРМНО-ИНВАРИАНТНОГО СКАЛЯРНОГО ПОЛЯ ПРИ $d=2$}

Конформно-инвариантной двумерной моделью с нетривиальной динамикой является теория Лиувилля с плотностью лагранжиана

$$
\mathcal{L}_{0}^{\text {Liouville }}=\frac{1}{2} \eta^{\mu \nu} \partial_{\mu} \psi \partial_{\nu} \psi-\frac{m^{2}}{\beta^{2}} e^{\beta \psi}
$$

Преобразования конформной симметрии действуют аффинным образом, так что экспоненциальное взаимодействие остается инвариантным:

$$
\delta \psi=f^{\alpha} \partial_{\alpha} \psi+\frac{1}{\beta} \partial_{\alpha} f^{\alpha}
$$

Канонический тензор энергии-импульса

$$
\theta_{\mu \nu}^{\text {canonical }}=\partial_{\mu} \psi \partial_{\nu} \psi-\eta_{\mu \nu}\left(\frac{1}{2} \eta^{\alpha \beta} \partial_{\alpha} \psi \partial_{\beta} \psi-\frac{m^{2}}{\beta^{2}} e^{\beta \psi}\right)
$$

снова не является бесследовым, $\eta_{\mu \nu} \theta_{\mu \nu}^{\text {canonical }} \neq 0$, однако после улучшения он приобретает это свойство:

$$
\theta_{\mu \nu}=\theta_{\mu \nu}^{\text {canonical }}+\frac{2}{\beta}\left(\eta_{\mu \nu} \square-\partial_{\mu} \partial_{\nu}\right) \psi, \quad \eta^{\mu \nu} \theta_{\mu \nu}=0
$$


Тензор $\theta_{\mu \nu}^{\text {canonical }}$ снова возникает вариационным образом, когда лиувиллева лагранжева плотность минимально расширяется с помощью произвольного метрического тензора. Аналогично мы получаем улучшенный тензор (3.4), вводя неминимальное взаимодействие:

$$
\begin{aligned}
& \mathcal{L}^{\text {Liouville }}=\frac{1}{\beta} R \psi+\frac{1}{2} g^{\mu \nu} \partial_{\mu} \psi \partial_{\nu} \psi-\frac{m^{2}}{\beta^{2}} e^{\beta \psi} \\
& T_{\mu \nu}=\frac{2}{\sqrt{|g|}} \frac{\delta}{\delta g^{\mu \nu}} \int \sqrt{|g|} \mathcal{L}^{\text {Liouville }}= \\
&=\partial_{\mu} \psi \partial_{\nu} \psi-g_{\mu \nu}\left(\frac{1}{2} g^{\alpha \beta} \partial_{\alpha} \psi \partial_{\beta} \psi-\frac{m^{2}}{\beta^{2}} e^{\beta \psi}\right)+\frac{2}{\beta}\left(g_{\mu \nu} D^{2}-D_{\mu} D_{\nu}\right) \psi \\
& T_{\mu \nu} \underset{g_{\mu \nu} \rightarrow \eta_{\mu \nu}}{\longrightarrow} \theta_{\mu \nu} .
\end{aligned}
$$

Тензор $T_{\mu \nu}$ в искривленном пространстве не является бесследовым,

$$
g^{\mu \nu} T_{\mu \nu}=\frac{2}{\beta^{2}} R \neq 0
$$

он становится бесследовым только в пределе плоского пространства, когда $R$ обращается в нуль. Соответственно действие, связанное с выражением (3.5), не инвариантно относительно преобразований Вейля, которые для скалярного поля $\psi$ принимают вид

$$
\psi \underset{\mathrm{Weyl}}{\longrightarrow} \psi-\frac{2}{\beta} \sigma .
$$

Такой вид преобразований необходим для инвариантности плотности взаимодействия $\sqrt{|g|} e^{\beta \psi}$. Однако кинетический член вместе с неминимальным членом не инвариантны, так что

$$
I^{\text {Liouville }}=\int \sqrt{|g|} \mathcal{L}^{\text {Liouville }} \underset{\text { Weyl }}{\longrightarrow} I^{\text {Liouville }}-\frac{2}{\beta^{2}} \int \sqrt{|g|}\left(R \sigma+g^{\mu \nu} \partial_{\mu} \sigma \partial_{\nu} \sigma\right) .
$$

Заметим, что вариация действия (последнее слагаемое в выражении (3.10)) не зависит от $\psi$. Поэтому уравнение для поля

$$
D^{2} \psi+\frac{m^{2}}{\beta} e^{\beta \psi}-\frac{1}{\beta} R=0
$$

обладает симметрией Вейля, хотя действие ей не обладает.

\section{4. ВЫВОД ТЕОРИИ ЛИУВИЛЛЯ ПРИ $d=2$ ИЗ ВЕЙЛЬ-ИНВАРИАНТНЫХ ТЕОРИЙ ПРИ $d>2$}

Мы видим, что в двумерном случае имеются существенные отличия от того, что мы получили при $d>2$ : в теориях, соответствующих последнему случаю, существует инвариантный относительно преобразования Вейля предшественник с бесследовым тензором энергии-импульса в искривленном пространстве, который приводит 
к бесследовому тензору энергии-импульса в плоском пространстве. При $d=2$ такой предшественник не является вейль-инвариантным, и тензор энергии-импульса становится бесследовым только в пределе плоского пространства.

Чтобы лучше понять двумерное поведение, построим предельную процедуру, которая приводит инвариантные относительно преобразования Вейля при $d>2$ модели (2.5) к двумерному случаю. Тем самым мы выделим шаги, на которых теряется вейль-инвариантность.

Чтобы в случае $d=2$ вывести теорию Лиувилля (3.5) из вейль-инвариантных моделей с полиномиальным взаимодействием (2.5) при $d>2$, положим

$$
\varphi=\frac{2 d}{\beta(d-2)} e^{(\beta(d-2) /(2 d)) \psi}
$$

и возьмем предел $d \rightarrow 2$, как и выше. Исследуем каждый из трех членов в выражении (2.5) по отдельности.

Для самодействия имеем

$$
\lambda \varphi^{2 d /(d-2)}=\lambda\left(\frac{2 d}{\beta(d-2)}\right)^{2 d /(d-2)} e^{\beta \psi} \underset{d \downarrow 2}{\longrightarrow} \frac{m^{2}}{\beta^{2}} e^{\beta \psi} .
$$

На последнем шаге для того чтобы избавиться от сингулярного множителя, мы перенормировали константу $\lambda$, введя величину $m^{2} / \beta^{2}$. Для кинетического члена взятие предела не вызывает затруднений:

$$
\frac{1}{2} g^{\mu \nu} \partial_{\mu} \varphi \partial_{\nu} \varphi \underset{d \downarrow 2}{\longrightarrow} \frac{1}{2} g^{\mu \nu} \partial_{\mu} \psi \partial_{\nu} \psi
$$

Однако неминимальный член не имеет предела, поэтому мы раскладываем экспоненту:

$$
\begin{aligned}
\frac{d-2}{8(d-1)} R \varphi^{2} & =\frac{d^{2}}{2 \beta^{2}(d-1)(d-2)} R e^{(\beta(d-2) / d) \psi}= \\
& =\frac{d^{2}}{2 \beta^{2}(d-1)(d-2)} R+\frac{d}{2 \beta(d-1)} R \psi+\cdots .
\end{aligned}
$$

В пределе $d=2$ выражения (4.2) и (4.3) и последний член в правой части формулы (4.4) приводят к лиувиллевой плотности лагранжиана (3.5) в искривленном пространстве. Первый член в правой части выражения (4.4) дает неопределенный результат в выражении для действия:

$$
\left.\int \sqrt{|g|} \mathcal{L}\right|_{d>2} \underset{d \downarrow 2}{\longrightarrow} \int \sqrt{|g|} \mathcal{L}^{\text {Liouville }}+\frac{2}{\beta^{2}(d-2)} \int \sqrt{|g|} R .
$$

Неопределенность возникает из-за того, что в размерности два величина $\sqrt{|g|} R$ представляет собой эйлерову плотность, а ее интеграл - поверхностный член, которые эффективно обращаются в нуль, коль скоро мы интересуемся объемными 
свойствами. Поэтому второе слагаемое в выражении (4.5) дает $0 / 0$ при $d=2$. Очевидно, модель Лиувилля воспроизводится, когда 0/0 интерпретируется как 0, но это приводит к потере вейль-инвариантности. Для поддержания вейль-инвариантности в пределе $d \downarrow 2$ нам следует тщательно вычислить не зависящую от $\psi$ величину $\int \sqrt{|g|} R /(d-2)$, т.е. нам требуется нечто вроде правила Лопиталя для размерной редукции.

Оказывается, что точную оценку величины $\int \sqrt{|g|} R /(d-2)$ в пределе $d \downarrow 2$ можно найти, обратившись к исходным идеям Вейля.

Прежде чем их описывать, заметим, что правила конформного преобразования (3.2) и преобразования Вейля (3.9) для $\psi$ получаются правильным образом, если подставить выражение (4.1) в соответствующие правила (2.2), (2.9б) для $\varphi$ и перейти к пределу $d \downarrow 2$. Такая же связь существует между уравнениями движения (3.11) и (2.8). Однако из редукции тензора энергии-импульса (2.6) для $\varphi$ получается тензор (3.6) для $\psi$ плюс слагаемое $\left(4 / \beta^{2}\right) G_{\mu \nu} /(d-2)$, которое не определено при $d=2$, поскольку как числитель, так и знаменатель обращаются в нуль. Отметим, что взятие следа от этой величины до предельного перехода $d \downarrow 2$ приводит к тому, что остается величина $\left(4 / \beta^{2}\right)(1-d / 2) R /(d-2)=-\left(2 / \beta^{2}\right) R$, которая сокращает не обращающийся в нуль след лиувиллевского тензора энергии-импульса. Это снова указывает на неопределенность как на причину отсутствия вейль-инвариантности.

\section{5. ВЕЙЛЕВСКАЯ ВЕЙЛЬ-ИНВАРИАНТНОСТЬ}

Чтобы получить определенное поведение величины $\int \sqrt{|g|} R /(d-2)$ в пределе $d \downarrow 2$, еще раз исследуем свойства преобразования Вейля кинетического члена для теории скалярного поля в размерности $d$ (самодействие инвариантно относительно преобразования Вейля и не требует дальнейшего обсуждения). Как уже отмечалось, кинетический член не является вейль-инвариантным, и это компенсируется неминимальным взаимодействием, приводящим к инвариантному относительно преобразований Вейля кинетическому действию

$$
I^{\text {kinetic }}=\int \sqrt{|g|}\left(\frac{1}{2} g^{\mu \nu} \partial_{\mu} \varphi \partial_{\nu} \varphi+\frac{d-2}{8(d-1)} R \varphi^{2}\right) .
$$

Однако Вейль предложил другой механизм для построения вейль-инвариантного кинетического члена: вместо использования неминимального взаимодействия он ввел “калибровочный потенциал" $W_{\mu}$, для того чтобы устранить невариантность [3]. Можно проверить, что действие

$$
I^{\mathrm{Weyl}}=\int \sqrt{|g|}\left(\frac{1}{2} g^{\mu \nu}\left[\partial_{\mu} \varphi+(d-2) W_{\mu} \varphi\right]\left[\partial_{\nu} \varphi+(d-2) W_{\nu} \varphi\right]\right)
$$

инвариантно относительно преобразований $(2.9)$, если $W_{\mu}$ преобразуется как

$$
W_{\mu} \underset{\text { Weyl }}{\longrightarrow} W_{\mu}-\frac{1}{2} \partial_{\mu} \sigma .
$$


Теперь потребуем, чтобы величина $I^{\text {kinetic }}$ в выражении (5.1) совпадала с величиной $I^{\text {Weyl }}$ в выражении (5.2). Этого можно достичь, если выполнено равенство

$$
\frac{R}{4(d-1)}=D^{\mu} W_{\mu}+(d-2) g^{\mu \nu} W_{\mu} W_{\nu}
$$

Это занятное уравнение типа уравнения Риккати знакомо в случае $d=2$, где из него следует, что $\sqrt{|g|} R$ является полной производной, обобщением этого условия на произвольные $d>2$ и является (5.4).

Прежде чем переходить к пределу $d \downarrow 2$, вычислим с помощью выражения (5.4) неоднозначный вклад в действие - последний член в выражении (4.5). Из (5.4) имеем

$$
\frac{\int \sqrt{|g|} R}{4(d-1)(d-2)}=\frac{1}{d-2} \int \partial_{\mu}\left(\sqrt{|g|} W^{\mu}\right)+\int \sqrt{|g|} g^{\mu \nu} W_{\mu} W_{\nu} .
$$

Первое слагаемое в правой части равенства не дает вклада даже при $d \neq 2$, поскольку подынтегральное выражение является полной производной при любых $d$, тогда как из второго возникает

$$
\lim _{d \downarrow 2} \frac{\int \sqrt{|g|} R}{d-2}=4 \int \sqrt{|g|} g^{\mu \nu} w_{\mu} w_{\nu}
$$

где $\left.w_{\mu} \equiv W_{\mu}\right|_{d=2}$ удовлетворяет согласно (4.4) уравнению

$$
4 D^{\mu} w_{\mu}=R \quad \text { при } \quad d=2 .
$$

Отметим, что выражения (5.3) и (5.7) согласованы с формулой преобразований Вейля для $R$ при $d=2: R \rightarrow e^{-2 \sigma}\left(R-2 D^{2} \sigma\right)$.

Таким образом, чтобы добиться вейль-инвариантности, следует дополнить действие зависящим от метрики, но не зависящим от $\psi$ членом

$$
\Delta I=\frac{8}{\beta^{2}} \int \sqrt{|g|} g^{\mu \nu} w_{\mu} w_{\nu}
$$

Согласно (5.3) и (5.7) вариация Вейля величины $\Delta I$ есть

$$
\Delta I \rightarrow \Delta I+\frac{2}{\beta^{2}} \int \sqrt{|g|}\left(R \sigma+g^{\mu \nu} \partial_{\mu} \sigma \partial_{\nu} \sigma\right)
$$

Это сокращает вейль-неинвариантность величины $I^{\text {Liouville }}$, см. (3.10).

Остается найти $w_{\mu}$, решив уравнение (5.7). Мы, конечно, интересуемся локальным решением, для того чтобы инвариантное относительно преобразований Вейля действие Лиувилля было локальным. Такое решение было найдено в работе [4]. Оно имеет вид

$$
w^{\mu}=\frac{\varepsilon^{\mu \nu}}{4 \sqrt{|g|}}\left(\frac{\varepsilon^{\alpha \beta}}{\sqrt{|g|}} \partial_{\alpha} g_{\beta \nu}+(\operatorname{ch} \omega-1) \partial_{\nu} \gamma\right) .
$$


Второе слагаемое в скобках представляет собой каноническую $S L(2, R)$ 1-форму, где

$$
\operatorname{ch} w=\frac{g_{+-}}{\sqrt{|g|}}, \quad e^{\gamma}=\sqrt{\frac{g_{++}}{g_{--}}}
$$

(индексы $(+,-)$ относятся к компонентам светового конуса $\left.\left(x^{0} \pm x^{1}\right) / \sqrt{2}\right)$. Соответствующая часть $w^{\mu}$ является вейль-инвариантной, а оставшаяся часть удовлетворяет закону преобразования (5.3). Решение (5.10) не единственно. Можно добавить к (5.10) любое инвариантное относительно преобразований Вейля слагаемое вида $\left(\varepsilon^{\mu \nu} / \sqrt{|g|}\right) \partial_{\nu} X$, поскольку оно не дает вклада в $(5.7)$.

Замечательно, что $w^{\mu}$ в выражении (5.10) не является контравариантным вектором, хотя $D_{\mu} w^{\mu}$ и является скаляром $R / 4$. Следовательно, наше вейль-инвариантное действие Лиувилля $I^{\text {Liouville }}+\Delta I$ не является инвариантным относительно диффеоморфизмов. Его $g^{\mu \nu}$-вариация определяет бесследовый тензор энергии-импульса, который, однако, не является (ковариантно) сохраняющимся.

Мы не знаем, как к этому относиться. Возможно, упомянутую выше неоднозначность можно использовать для избавления от неинвариантности относительно диффеоморфизмов, но нам это не удалось. Поэтому представляется, что локальное действие Лиувилля в искривленном пространстве может быть инвариантным относительно диффеоморфизмов или относительно преобразований Вейля, но не относительно их обоих.

Если это предположение верно, то мы сталкиваемся с "аномальной" ситуацией в классической теории поля, которая ранее встречалась только в квантованной теории поля. Известно, что в размерности два инвариантная относительно диффеоморфизмов лагранжева плотность $(\sqrt{|g|} / 2) g^{\mu \nu} \partial_{\mu} \varphi \partial_{\nu} \varphi$ также инвариантна относительно преобразований Вейля, которые преобразуют метрический тензор, но не преобразуют скалярное поле $\varphi$ (т.е. уравнение $(2.9)$ при $d=2$ ). Однако эффективное квантовое действие, которое получается путем взятия функционального интеграла по $\varphi$, дает метрическое выражение, являющееся инвариантным относительно диффеоморфизмов или относительно преобразований Вейля, но не относительно их обоих [5].

Если отказаться от локальности, можно легко построить ковариантное решение для $w_{\mu}$ в виде $\partial_{\mu} w$, где $w$ изменяется при преобразовании Вейля как (cp. (5.3))

$$
w \rightarrow w-\frac{\sigma}{2} .
$$

Очевидно, что

$$
\begin{gathered}
D^{2} w=\frac{R}{4}, \\
w(x)=\frac{1}{4} \int d^{2} y \sqrt{|g(y)|} \frac{1}{D^{2}(x, y)} R(y),
\end{gathered}
$$

где функция Грина определяется как решение следующего уравнения:

$$
D_{x}^{2} \frac{1}{D^{2}(x, y)}=\frac{1}{\sqrt{|g|}} \delta^{2}(x-y) .
$$


Уравнение (5.12) выполнено в силу (5.13), и добавление действия Лиувилля представляет собой не что иное, как действие Полякова [5]:

$$
\Delta I=\frac{1}{2 \beta^{2}} \int \partial^{2} x d^{2} y \sqrt{|g(x)|} R(x) \frac{1}{D^{2}(x, y)} \sqrt{|g(y)|} R(y) .
$$

Это дает действие для теории Лиувилля, инвариантное относительно диффеоморфизмов и преобразований Вейля, которое, однако, является нелокальным. Вопрос о том, можно ли добиться еще и локальности, остается открытым.

\section{Список литературы}

[1] C. Callan, S. Coleman, R. Jackiw, Ann. Phys., 59 (1970), 42.

[2] B. Zumino, "Effective Lagrangians and Broken Symmetries", Lectures on Elementary Particles and Quantum Field Theory 2 (2006), Brandeis Univ. Summer Lectures, 1970, eds. S. Deser, M. Grisaru, H. Pendleton, MIT, Cambridge MA, 1971, 437.

[3] A. Iorio, L. O'Raifeartaigh, I. Sachs, C. Wiesendanger, Nucl. Phys. B, 495 (1997), 433.

[4] S. Deser, R. Jackiw, Int. J. Mod. Phys. B, 10 (1996), 1499.

[5] А.М. Поляков, Калибровочные поля и струны, Удмуртск. унив-т, Ижевск, 1999. 